\title{
STRONG AND ORDINARY SUMMABILITY
}

\author{
GEORGE G. LORENTZ AND KARL ZELLER ${ }^{1)}$
}

(Received June 28, 1963)

1. Introduction. We consider infinite matrices $A=\left(a_{n k}\right)$ and corresponding matrix transforms and summability methods (compare [5]). A sequence $\left\{s_{k}\right\}$ is said to be $\bar{A}$-summable to the value $\sigma$, if all sums

$$
\sigma_{n}=\sum_{k=0}^{\infty} a_{n k} s_{k}, n=0,1, \cdots
$$

exist and converge to $\sigma$ for $k \rightarrow \infty$. The sequence $\left\{s_{k}\right\}$ is strongly $A$-summable (shortly: $\bar{A}$-summable) to the value $\sigma$, if all sums

$$
\sigma_{n}=\sum_{k=0}^{\infty} a_{n k}\left|s_{k}-\sigma\right|, \quad n=0,1, \cdots
$$

exist and converge to zero. Strong summability is usually considered only for positive $A$ (i.e., for $a_{n k} \geqq 0$ ). In this case the limit $\sigma$ is uniquely determined [3] if $A$ is regular, i.e., sums each convergent sequence to its ordinary limit. A row-finite matrix contains only a finite number of non-zero elements in each row; a normal matrix has non-zero elements on the main diagonal and zeros above it.

We compare here strong and ordinary summability methods. The basic question is the following. Given a matrix $A$, does there exist a matrix $\mathrm{B}$, such that a sequence $\left\{s_{k}\right\}$ is $B$-summable if and only if it is strongly $A$-summable? (In this case $B$ and $\bar{A}$ are called equivalent). For the Cesàro method of order one, $A=C_{1}$, the question has been answered positively in [4]. We generalize this result to arbitrary row-finite regular matrices $A$ (Theorem 1 ). There exist, however, row-infinite regular matrices $A$ for which no equivalent $B$ exists (Theorem 4). Even for row-finite regular $A$ it is not always possible to find a normal $B$ equivalent to $\bar{A}$. We give (Theorem 2) necessary and sufficient conditions for the existence of a matrix $B$ with these properties. As a simple special case of Theorem 2 we have: the method $\bar{A}$ is not equivalent to any normal ordinary method $B$ if $\rho_{k}=\max _{n} a_{n k} \rightarrow 0$ as $k \rightarrow \infty$. A corollary (Theorem 3 ) of Theorems 1 and 2 concerns the question of equivalence of ordinary row-finite and normal methods.

We avoid the use of Functional Analysis (although its application could

1) This work has been supported by the Office of Scientific Research, U.S. Air Force, through the Grant no. AF-AFOSR-62-138. 
shorten some proofs). For simplicity we assume that the matrix $A$ of the strong summability method is always regular and positive; generalizations are possible.

2. Row-finite matrices. A strong summability method $\bar{A}$, based on a row-finite regular matrix $A$, can always be replaced by an ordinary matrix method:

THEOREM 1. For each row-finite regular positive matrix $A$ there is a row-finite regular positive matrix $B$ such that a sequence $\left\{s_{k}\right\}$ is $\bar{A}$-summable to the value $\sigma$ if and only if it is B-summable to this value.

The proof is based on

LEMMA 1. Let $K$ be a finite set of natural numbers, let $x_{k}$ be complex and $a_{k}$ positive $\left(a_{k} \geqq 0\right)$ values defined for $k \in K$. Put

$$
\sum_{k \in K}\left|x_{k}\right|=x, \quad \sum_{k \in R} a_{k}=a .
$$

Then there exists a subset $K^{\prime}$ of $K$ such that

$$
\left|\sum_{k \in K^{\prime}} x_{k}\right| \geqq \frac{1}{6} x, \quad \sum_{k \in K^{\prime}} a_{k} \geqq \frac{1}{2} a .
$$

ProOF. One of the sums $\sum\left|\operatorname{Re} x_{k}\right|$ or $\sum\left|\operatorname{Im} x_{k}\right|$ is not less than $\frac{1}{2} x$, hence it is sufficient to derive (4) from (3) for the case of real $x_{k}$ but with $\frac{1}{6} x$ replaced by $\frac{1}{3} x$. For real $x_{k}$, we destinguish two cases. If $\left|\sum_{k \in B} x_{k}\right| \geqq \frac{1}{3} x$, we can take $K^{\prime}=K$. If this absolute value is less than $\frac{1}{3} x$, let $K^{+}, K^{-}$denote the sets of $k$ with $x_{k} \geqq 0$ or $x_{k}<0$, respectively. Then we select $K^{\prime}$ equal to one of the sets $K^{+}$, $K^{-}$, so as to satisfy the second condition (4); we will also have $\left|\sum_{k \in K^{\prime}} x_{k}\right| \geqq \frac{1}{3} x$

PROOF OF THEOREM 1. It is obviously sufficient to find a regular matrix $B$ such that $\bar{A}-\lim s_{n}=0$ and $B$-lim $s_{n}=0$ are equivalent.

For each $n=0,1, \cdots$, let $K=K_{n}$ be the finite set of integers $k$ for which $a_{n k}>0, k \in K, a_{n k}=0, k \notin K$. If $\sum_{k} a_{n k}=a_{n}$, we consider all subsets $K^{\prime}=K_{n v}^{\prime}$, $\nu=1,2, \cdots, N(n)$ of $K_{n}$ which have the property $\sum_{k \in K^{\prime}} a_{n k} \geqq \frac{1}{2} a_{n}$. Corresponding to one row $a_{n k}$ of $A$, let us define $N(n)$ rows of a matrix $B$ (each corresponding to a set $K_{n v}^{\prime}$ ) which consist of the numbers 
(5) $b_{m k}=\left(\sum_{k \in K^{\prime}} a_{n k}\right)^{-1} a_{n k}$ if $k \in K_{n v}^{\prime}, b_{m k}=0$ if $k \notin K_{n v}^{\prime}$.

Since $A$ is regular, for some $M>0, a_{n} \leqq M$, and hence $a_{n k} \leqq M b_{m k}$. We order the rows of $B$ in the following way: first $N(0)$ rows corresponding to the row $a_{0 k}$ of $A$; then $N(1)$ rows corresponding to the row $a_{1 k}$ of $A$; and so on.

It is easy to see that $B$ is regular, and that $\sum b_{n k} s_{k}$ converges to zero whenever the sequence $s_{n}$ has the property $\sum_{k \in B_{n}} a_{n k}\left|s_{k}\right| \rightarrow 0$. Conversely, if $s_{k}$ is $B$-summable to zero, then taking $x_{k}=a_{n k} s_{k}, a_{k}=a_{n k}$ in Lemma 1, we see that for at least one $m$ with the corresponding set $K_{n v}^{\prime}$,

$$
\sum_{k \in X_{n}} a_{n k}\left|s_{k}\right| \leqq 6\left|\sum_{k \in K^{\prime} n v} a_{n k} s_{k}\right| \leqq 6 M\left|\sum_{k} b_{m k} s_{k}\right| .
$$

Thus, $s_{n}$ is $\bar{A}$-summable to zero, and the result follows.

3. Normal matrices. In contrast to Theorem 1, it is not always possible to replace a row-finite strong summability method by a normal matrix method. We prove more. We consider also row-infinite matrices, and give necessary and sufficient conditions when this replacement is possible.

For a regular positive matrix $A$ we write

$$
\rho_{k}=\max _{n} a_{n k}, k=0,1, \cdots .
$$

THEOREM 2. Let $A$ be a regular positive matrix. There exists a normal method $B$ which is equivalent to $\bar{A}$ if and only if for some $M$ and $k_{0}$,

$$
\begin{aligned}
& \rho_{k} \neq 0, \quad k \geqq k_{0}, \\
& \sum_{k=k_{0}}^{\infty} a_{n k} \rho_{k}{ }^{-1} \leqq M, n=0,1, \cdots .
\end{aligned}
$$

If the conditions are satisfied, $B$ may be taken to be regular and consistent with $\bar{A}$.

The following two lemmas will be needed:

LEMMA 2. Let $\rho_{k} \geqq 0, k=0,1, \cdots$ be an arbitrary sequence and $B$ be an arbitrary matrix method. Then (i) $B$ sums all sequences $\left\{s_{k}\right\}$ with $\rho_{k} s_{k} \rightarrow 0$ if and only if

$$
\lim _{n \rightarrow \infty} b_{n k} \quad \text { exists for each } k=0,1, \cdots,
$$

and there are $M$ and $k_{0}$ such that 


$$
\begin{gathered}
\rho_{k}=0, k \geqq k_{0} \text { implies } b_{n k}=0, n=0,1, \cdots, \\
\sum_{\rho_{k} \neq 0}\left|b_{n k}\right| \rho_{k}^{-1} \leqq M, n=0,1, \cdots
\end{gathered}
$$

(ii) $B$ sums all sequences $\left\{s_{k}\right\}$ with $\sum \rho_{k}\left|s_{k}\right|<+\infty$ if and only if $B$ satisfies (10) and there are $M$ and $k_{0}$ such that

$$
\left|b_{n k}\right| \leqq M \rho_{k}, \quad k \geqq k_{0} .
$$

ProOF. In both cases (i), (ii), it is easy to prove the necessity of the existence of a $k_{0}$ for which (11) is satisfied. If this holds, we can omit from $B$ all columns $b_{n k}$ for which $\rho_{k}=0$. Thus it is sufficient to prove our lemma for the case when $\rho_{k}>0, k=0,1, \cdots$. But then the lemma follows from the wellknown theorems about matrices which sum all null sequences, or all absolutely convergent series (compare for example [1, p. 63] for (i), and [2,p.29] for (ii)).

LEMMA 3. Let $A$ be a positive regular matrix, with the $\rho_{k}$ defined by (7). Then $\bar{A}-\lim s_{n}=0$ impies $\rho_{k} s_{k} \rightarrow 0$.

Proof. Assume that $\left\{s_{n}\right\}$ is $\bar{A}$-summable to zero, we have to prove that $\rho_{k} s_{k} \rightarrow 0$. Let $\epsilon>0$ be arbitrary. We take first $N$ so large that $\sum_{k} a_{n k}\left|s_{k}\right|<\epsilon$ for $n \geqq N$ and then $K$ so large that $a_{n k}\left|s_{k}\right|<\epsilon$ for $k \geqq K, n<N$. Then $a_{n k}\left|s_{k}\right|<\epsilon$ for $k \geqq K$ and all $n$, hence $\rho_{k}\left|s_{k}\right|<\epsilon, k \geqq K$.

Proof of The Sufficency of the Conditions. Assume that the conditions of Theorem 2 are satisfied. We may suppose that $k_{0}=0$. Since $A$ is regular, the sequence $\rho_{k}$ is bounded. We cannot have $\rho_{k} \rightarrow 0$, since then (9) would imply that the sequence $\{1,1, \cdots\}$ is $A$-summable to zero, in contradiction to the regularity. Hence we can find a sequence $k_{j}$ strictly increasing to infinity for which $\rho_{k_{j}} \rightarrow \rho \neq 0$. We define $B$ as follows:

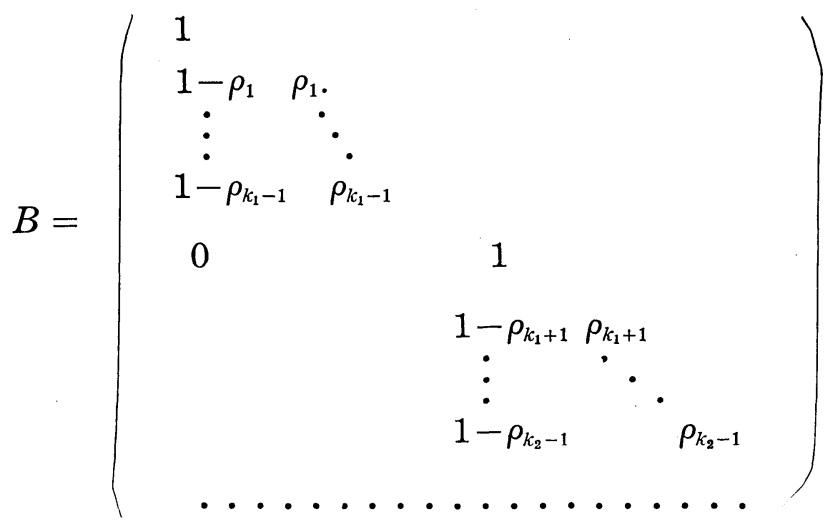


The matrix $B$ is regular, and one easily sees that $B$-lim $s_{n}=0$ is equivalent to $\rho_{n} s_{n} \rightarrow 0$. By Lemma 2 (i) applied to the matrix $A$, we have $A$-lim $s_{n}=0$, and hence even $\bar{A}-\lim s_{n}=0$ for all sequences $s_{n}$ with $\rho_{n} s_{n} \rightarrow 0$. By Lemma 3, $\bar{A}-\lim s_{n}=0$ is equivalent to $\rho_{n} s_{n} \rightarrow 0$. This, together with the regularity and the linearity of the methods $B$ and $A$, implies that $B$ - $\lim s_{n}=\sigma$ is equivalent to $\bar{A}-\lim s_{n}=\sigma$.

PROOF OF THE NECESSITY. We begin with the following

LEMMA 4. If a normal matrix $B$ and a sequence $\epsilon_{k}>0$ are given, there exists a sequence $\left\{s_{k}\right\}$ with the properties

$$
\left|b_{k k}\right|\left|s_{k}\right| \geqq \epsilon_{k},\left|\sigma_{n}\right|=\epsilon_{n},
$$

where $\sigma_{n}$ is the B-transform of $\left\{s_{k}\right\}$.

Making $\epsilon_{k} \rightarrow 0$ slowly, we obtain a sequence $s_{k}$ which is $B$-summable to zero, and whose terms in absolute value are close to $\left|b_{k k}\right|^{-1}$.

PROOF. We construct $s_{k}$ by induction. Put $s_{0}=\epsilon_{0} b_{00}{ }^{-1}$. If $s_{0}, \cdots, s_{k-1}$ are already determined, let $\tau_{k}=b_{k 0} s_{0}+\cdots+b_{k, k-1} s_{k-1}$. We choose $s_{k}$ so that the modulus of $b_{k k} s_{k}$ is $\left|\tau_{k}\right|+\epsilon_{k}$, and the sign is opposite to that of $\boldsymbol{\tau}_{k}$; the sequence $s_{k}$ satisfies (15).

Now we assume that there is a normal method $B$ equivalent to $\bar{A}$. From Lemma 2 (ii) we derive that each sequence $s_{k}$ with $\sum \rho_{k}\left|s_{k}\right|<+\infty$ is $\bar{A}$-summable to 0 . This applies also to $\left|s_{k}\right|$, hence $s_{k}$ is $\bar{A}$-summable to zero, and thus $B$-summable. Again from Lemma 2 (ii) we derive that

$$
\left|b_{k k}\right| \leqq M \rho_{k}, k \geqq k_{0} \text {. }
$$

Since $b_{k k} \neq 0$, we must have $\rho_{k} \neq 0, k \geqq k_{0}$, so that (8) is satisfied.

Applying Lemma 4 and (16), we find, for each null sequence $\epsilon_{k}>0$, a sequence $s_{k}, B$-summable to zero, for which $M \rho_{k}\left|s_{k}\right| \geqq \sqrt{\epsilon_{k}}$. Hence $s_{k}$ is $\bar{A}$ summable and the sequence $\epsilon_{k} \rho_{k}{ }^{-1}=o\left(\left|s_{k}\right|\right)$ is $\bar{A}$-summable to zero. Applying Lemma 2 (i) to the matrix with the coefficients $a_{n k} \rho_{k}{ }^{-1}$, we see that also the condition (9) is satisfied. This completes the proof of Theorem 2.

Theorems 1 and 2 contain the following corollary:

THEOREM 3. There exists a row-finite regular matrix $B$ which provides a 1-1 mapping and which is not equivalent to any normal matrix.

Proof. We take the matrix $B$ which corresponds to the strong $C_{1}$ - 
summability according to the proof of Theorem 1 . Since $B$ contains all rows of $C_{1}$, it provides a $1-1$ mapping as the latter matrix.

If the restriction to a 1-1 mapping is omitted, the construction of $B$ becomes trivial. In this case one can take for $B$ any row-finite regular matrix for which $b_{n k_{i}}=-b_{n, k_{t}+1}, n, i=0,1, \cdots$ for some sequence $k_{i} \rightarrow \infty$.

4. Row-infinite matrices. Another counterpart of Theorem 1 is the fact that a row-infinite strong summability method is in general not equivalent to an ordinary matrix method:

THEOREM 4. There exists a row-infinite regular positive matrix $A$ such that no ordinary matrix method $B$ sums exactly the strongly A-summable sequences.

PROOF. We put

$$
A=\left(\begin{array}{ccccccc}
2^{-0} & 0 & 2^{-1} & 0 & 2^{-2} & 0 & \ldots \\
0 & 1 & 0 & 0 & 0 & 0 & \ldots \\
0 & 0 & 0 & 1 & 0 & 0 & \ldots \\
0 & 0 & 0 & 0 & 0 & 1 & \ldots \\
\ldots & \ldots & \ldots & \ldots & \ldots & \ldots & \ldots
\end{array}\right) .
$$

The strongly $A$-summable sequences $\left\{s_{k}\right\}$ are exactly the sequences for which

$$
\sum_{k=0}^{\infty} 2^{-k}\left|s_{2 k}\right|<+\infty ; \quad \lim _{k \rightarrow \infty} s_{2 k-1} \text { exists. }
$$

If $B$ sums every such sequence, then the matrix $C$ :

$$
c_{n k}=2^{k} b_{n, 2 k}
$$

sums every sequence $\left\{w_{k}\right\}$ with $\sum\left|w_{k}\right|<+\infty$. The statement of the theorem is therefore a consequence of the following lemma :

LEMMA 5. If a matrix $C$ sums every sequence $\left\{w_{k}\right\}$ satisfying $\sum\left|w_{k}\right|<$ $+\infty$, then it sums also a sequence $\left\{x_{k}\right\}$ with $\sum\left|x_{k}\right|=+\infty$.

PROOF. If is easy to see (and is also the special case of Lemma 2(ii) when all $\rho_{k}=1$ ) that the assumption about $C$ of the lemma is equivalent to the following. There exists an $M \geqq 0$ and a (bounded) sequence $\left\{c_{k}\right\}$ such that

$$
\begin{aligned}
& \left|c_{n k}\right| \leqq M, \quad n, k=0,1, \cdots, \\
& \lim _{n \rightarrow \infty} c_{n k}=c_{k}, \quad k=0,1, \cdots .
\end{aligned}
$$


By means of these conditions we construct the required sequence $x_{k}$. We define recursively integers $k_{0}<l_{0}<k_{1}<l_{1}<\cdots$ and $n_{0}<n_{1}<\cdots$ such that:

$$
\begin{gathered}
\left|c_{k_{j}}-c_{l_{j}}\right| \leqq 2^{-j}, j=0,1, \cdots ; \\
\left|c_{n k_{j}}-c_{n l_{j}}\right| \leqq 2^{-j}, n \leqq n_{j} ; \\
\left|c_{n k_{j}}-c_{k_{j}}\right| \leqq 2^{-j},\left|c_{n l_{j}}-c_{l_{j}}\right| \leqq 2^{-j}, n>n_{j+1} .
\end{gathered}
$$

If $k_{j-1}, l_{j-1}, n_{j}$ are already determined, we extract a convergent subsequence from the bounded vector sequence $\left\{c_{k}, c_{0 k}, c_{1 k}, \cdots c_{n_{j} k}\right\}_{k=0, \infty=0}^{\infty}$ and hence are able to satisfy (21) and (22) with proper $k_{j}, l_{j}$; an integer $n_{j+1}$, suitable for (23), exists because of (20). From (23) and (21) we derive

$$
\left|\mathrm{c}_{n k_{j}}-c_{n l_{j}}\right| \leqq 3 \cdot 2^{-j}, n>n_{j+1} \text {. }
$$

Now we put

$$
x_{k_{j}}=-x_{l}=\frac{1}{j+1}, j=0,1, \cdots ; \quad x_{k}=0 \text { for other } k .
$$

The $C$-transform of $x_{k}$

$$
\sum_{k} c_{n k} x_{k}=\sum_{j=0}^{\infty}\left(c_{n k_{j}}-c_{n l_{j}}\right) \frac{1}{j+1}
$$

exists because of (22). Also,

$$
\sum_{j=0}^{\infty}\left|c_{n k_{j}}-c_{n l_{j}}\right| \leqq 3 \sum_{j=0}^{\infty} 2^{-j}+2 M,
$$

because of (22), (24) and (19). By a variant of Toeplitz' theorem ([1, p. 63]; this is the special case of Lemma 2(i) when all $\left.\rho_{k}=1\right)$, the matrix $D=\left(d_{n k}\right)$, $d_{n_{j}}=c_{n k_{j}}-c_{n l_{j}}$ sums all null sequences. Hence $\left\{x_{k}\right\}$ is $C$-summable, while $\sum\left|x_{k}\right|=+\infty$.

\section{REFERENCES}

[1] R.G. Cooke, Infinite matrices and sequence spaces, London, Macmillan and Co., 1950.

[2] H. HAHN, Über Folgen linearer Operationen, Monatshefte Math. Phys. 32(1922), 3-88.

[3] H. J. HAMilton and J. D. Hill, On strong summability, Amer. Journ. Math. 60 (1938), 588-594.

[4] K. ZELLER, Über die Darstellbarkeit von Limitierungsverfahren mittels Matrixtransformationen, Math. Zeitschr. 59(1953),271-277.

[5] K. ZELLER, Theorie der Limitierungsverfahren, Ergebnisse der Mathematik und ihrer Grenzgebiete, Springer-Verlag, Berlin, 1958. 